\title{
EFFECT OF APPLYING SURFACE AND BUBBLER IRRIGATION SYSTEMS ON FRUITFUL WASHINGTON NAVEL ORANGE TREES PRODUCTION
}

\author{
HUSSIEN, S.M. ${ }^{1}$, E.A. ISMAIL ${ }^{1}$, M. N. H. ISMAIL ${ }^{1}$ and T.A. EID ${ }^{2}$ \\ 1. Horticultural Research Inst. ARC, Giza, Egypt. \\ 2. Soils, Water and Enviro. Res. Inst., ARC, Giza, Egypt.
}

(Manuscript received 15 December 2013)

\begin{abstract}
A field experiment was conducted at a private farm at Giza in the seasons of 2011 and 2012 to study and evaluate water utilization efficiency, vegetative and roots growth parameters, fruiting measurements, fruit quality and leaf mineral content of Washington Navel orange trees under two irrigation systems. Trees under study aged 35 years budded on sour orange rootstock and grown on clayey loamy soil. Trees were surface irrigated since the farm initiation, then subjected to bubbler and surface irrigation systems in the recent study for comparison. The obtained results pointed out that, surface irrigation consumptive use was (7480 and $7310 \mathrm{~m}^{3} / \mathrm{fed}$. in the two seasons) followed by bubbler irrigation system (4302 and $3913 \mathrm{~m}^{3} / \mathrm{fed}$.). Water consumptive values increased as soil moisture maintained high by surface irrigation. Monthly water use was low after January and then increased to reach its maximum during June, July and August and then decreased again. On the other hand, bubbler irrigation system supported an increase in water utilization efficiency up to $59.4 \%$.

Bubbler irrigation system increased both vegetative and root growth parameters i.e., (shoot length, leaf area, root length, number of roots and dry weight). Moreover, bubbler irrigation system resulted in a significant increase in fruiting parameters (yield $\mathrm{kg}$ per tree, number of fruits/tree, fruit weight g.). In addition to that most of both physical and chemical studied fruit characteristics were improved by bubbler than the surface irrigation method during the first and second seasons. However, leaf mineral content ( $\mathrm{N}, \mathrm{P}$ and $\mathrm{K}$ ) was not significantly affected by both investigated irrigation methods in the two seasons of study.

Bubbler irrigation system results in more effective roots $(<2$ $\mathrm{mm}$ ) in length and number compared with the surface one. This increase was reflected in improved yield and quality of orange production.

In brief, it could be concluded and recommended for Washington Navel orange trees grown on clay loamy soil to change surface to bubbler irrigation system for saving more irrigation water with better fruit yield, fruit quality, system and increasing water utilization efficiency to $59.4 \%$.
\end{abstract}

\section{INTRODUCTION}

Citrus is one of the most important world fruit crops. However, citrus in Egypt is ranked the first in this respect. Water for agriculture in Egypt is becoming a major 
constraint therefore better water management can be carried out through the efficiency of modern irrigation systems. Water is mainly consumed in agricultural purposes in Egypt and in the world (approximately $80 \%$ ). The rate of water consumption for industrial and domestic needs is gradually increasing which necessitates more efficient use of available water resources. Consequently, irrigation systems with a contribution to water saving (drip irrigation system, etc.) should be used more. Surface irrigation systems have been used extensively in fruit growing, and transition to drip irrigation system has started and being preferred more in recent years. These new systems are capable of delivering water in controllable small quantities as after and as long as needed. On the other hand, maximizing the use of modern irrigation systems became essential according to the increase in water demand (Brown, 1999) especially in arid and semiarid regions as Egypt, where population is fast increasing. Because drip irrigation system offers certain advantages such as fruit quality, decreasing labour costs, saving irrigation water, etc., many fruit growers have adopted this method. The adoption of drip irrigation system has expanded further especially at the regions with limited water resources.

Water is essential for citrus trees (or for any plant) because it is an integral component of the biochemical reactions that occur within the plant. Water is also important as it transport plant nutrients and other substances throughout the tree. Water also helps maintain plant temperature through transpiration. Finally, water helps maintain leaf and fruit turgidity.

Water requirement differs considerably with season, soil type and tree variety. When a tree suffers from lack of water, its yield decreases even it may recover after irrigation. On the other hand, increasing the number of irrigations (or water quantity) may result in injuring the crop and the soil besides being a waste of water and labour.

During March - June, soil moisture promotes spring flush and increases canopy size. During the fruit development stage, soil moisture is required to increase fruit size, but during the later stages of fruit maturation too much soil moisture reduces the accumulation of sugars and increases juice acidity. Irrigation management is very important nowadays in Egyptian lands, due to the shortage in water resources, as well as the expansion of agriculture in the new lands. Therefore, it is necessary to determine the optimum amounts of water for fruit trees for maximum crop production. On sandy soils, Koo and Smajstrla (1985) showed that the greatest yields of citrus occurred when about $80 \%$ of the area under the tree canopy was irrigated. Abdel-Missih et al., (1977) concluded that vegetative growth of Washington naval orange trees and also the growth rate of the fruit were generally enhanced by the addition of more water. Severe water stress decreased yield and fruit quality. Irrigation at 2/3 available soil moisture gave the best results. Ali and Gobran (2002) concluded that irrigation when 50\% 
of available soil moisture is depleted combined with potassium spray application improves growing, flowering, fruiting and water use of Washington Novel orange trees.

The purpose of this study is to identify the effects of converting surface to bubbler irrigation system on orange fruit quality, vegetative growth, leaf mineral content, root system growth and water utilization efficiency.

\section{MATERIALS AND METHODS}

The present study was carried out in a private farm at Wardan, Giza governorate, Egypt on clayey loamy soil during the two growing seasons 2011 and 2012, respectively. The main target of this investigation is to study the effect of changing the existing surface irrigation method to Bubbler irrigation on vegetative growth, yield and fruit quality of Washington Navel orange trees budded on sour orange rootstock.

Trees were 35 years old planted at $6 \times 6$ meters apart. The experimental soil was analyzed, Table (1). Trees were subjected to standard fertilization, pruning and protection programs as recommended by the Ministry of Agriculture.

Table 1. Physical properties of the orchard soil.

\begin{tabular}{|c|c|c|c|c|}
\hline \multicolumn{4}{|c|}{ Parameter } & Value \\
\hline \multicolumn{5}{|c|}{ Particle size distribution (\%): } \\
\hline \multicolumn{2}{|r|}{ Clayey } & \multicolumn{2}{|l|}{$\%$} & 34.4 \\
\hline \multicolumn{2}{|r|}{ Silt } & \multicolumn{2}{|l|}{$\%$} & 56.4 \\
\hline \multicolumn{2}{|r|}{ Fine sand } & \multicolumn{2}{|l|}{$\%$} & 8.20 \\
\hline \multicolumn{2}{|r|}{ Coarse sand } & $\%$ & & 1.0 \\
\hline \multicolumn{3}{|c|}{ Texture class } & \multicolumn{2}{|c|}{ Clayey loam } \\
\hline \multicolumn{5}{|c|}{ Water parameters and bulk density } \\
\hline Depth & $\begin{array}{c}\text { Field capacity (FC) } \\
\%(\mathrm{w} / \mathrm{w})\end{array}$ & $\begin{array}{l}\text { Wilting Point } \\
\text { (WP) \% (w/w) }\end{array}$ & $\begin{array}{l}\text { Available wat } \\
\text { (AW) \% (w/v }\end{array}$ & $\begin{array}{l}\text { Bulk density } \\
\text { (BD) } \mathrm{gm} . / \mathrm{cm}^{3}\end{array}$ \\
\hline $0-15$ & 39.80 & 18.62 & 21.18 & 1.15 \\
\hline $15-30$ & 33.71 & 17.48 & 16.23 & 1.24 \\
\hline $30-45$ & 30.91 & 16.91 & 13.28 & 1.21 \\
\hline $45-60$ & 29.12 & 16.50 & 12.62 & 1.28 \\
\hline
\end{tabular}

Irrigation treatments used in this study were as follows:

1- Surface irrigation system.

2- Bubbler irrigation system.

Twenty seven trees (in three replicates) were devoted for each irrigation system (nine trees/replicate).

\section{Irrigation water applied (IWA) of surface irrigation system:}

Irrigation water was calculated by the summation of the daily records of class $\mathrm{A}$ pan evaporation. Submerged flow orifice with fixed dimension was used to measure the amount of water applied (Michael, 1978). 


\section{Amount of applied irrigation water (AIW) of bubbler irrigation system:}

Potential evapotranspiration (ETp) values were obtained by class A pan (Doorenbos and Priutt, 1984):

Each tree line is served by two lateral lines about $200 \mathrm{~cm}$ apart (i.e., $100 \mathrm{~cm}$ from each side of the pseudo stems). Lateral lines equipped with build-in bubblers of $60 \mathrm{l} / \mathrm{h}$ discharge were spaced $3 \mathrm{~m}$ apart on the 2 bubblers.

\section{The following measurements were recorded:}

A- Growth parameters: shoot length, number of leaves/shoot and leaf area at mid August of both studied seasons.

\section{B- Yield and fruit quality.}

At harvest time, yield of each tree was recorded as number of fruits and $\mathrm{kg}$. / tree. Sample of ten fruits from each tree of each treatment was taken to study:

1- Physical fruit properties: fruit weight, size, rind thickness and juice weight percentage.

2- Chemical fruit properties, acidity (\%), ascorbic acid ( $\mathrm{mg} / 100 \mathrm{~g}$ juice) according to (A.O.A.C, 1990), total soluble solids (\%) using hand refractometer and total soluble/acid ratio were calculated.

3. Water utilization efficiency (WUE) values were calculated according to Jensen (1983) as follows:

Fruits yield $(\mathrm{kg}) /$ feddan

WUE $=$

$$
\text { Amount of applied irrigation water }\left(\mathrm{m}^{3} /\right. \text { fed.) }
$$

C- Leaf nutrient analysis included $\mathrm{N}$ by the micro-Kjeldahl digestion method as described by A.O.A.C. (1990) and K using wet digestion using the Flame photometer for determination. Total phosphorus content was determined using a Spekol spectrophotometer at 882.0 uv according to the method described by Evenhuis, (1978).

\section{D- Root study:}

Soil samples were taken on December (2012) only at 0-30, 30-60 and 60-90 $\mathrm{cm}$ depth at $100 \mathrm{~cm}, 200 \mathrm{~cm}$ and $300 \mathrm{~cm}$ from the tree trunk in the four directions. Root length ( $<2 \mathrm{~mm}$ and $>2 \mathrm{~mm}$ root thick) was assessed $(\mathrm{cm})$ and root dry weight (g.)/hole (1750.8 $\mathrm{cm}^{3}$ or $1.628 \mathrm{~kg}$ soil) according to Cahoon et al., (1959) and Ford (1962).

\section{Statistical analysis:}

All the obtained data during the two seasons of the study were subjected to analysis of variance method according to Snedecor and Cochran (1990). Meanwhile, differences between means were compared using Duncan's multiple range test at $5 \%$ level (Duncan, 1955). 


\section{RESULTS}

\section{A- Orange trees water relations parameters:}

\section{A-1. Applied irrigation water:}

Seasonal applied irrigation water for orange trees decreased under bubbler irrigation as compared with surface irrigation in both seasons. As it reached (4302 and $3913 \mathrm{~m}^{3} / \mathrm{fed}$.) in bubbler system and (7480 and $7310 \mathrm{~m}^{3} / \mathrm{fed}$.) in the surface one, respectively (Table 2 ). Such results might be reasonable, since more frequent irrigation period provide high evaporation opportunity from surface irrigation rather than bubbler irrigation. The seasonal water use values were obtained from the sum of water consumptive use for all irrigations per treatment, divided by the irrigation efficiency from January until December in each season.

Table 2. Monthly and seasonal applied irrigation water to orange trees by irrigation system in 2011 and 2012 growing seasons.

\begin{tabular}{|c|c|c|c|c|c|c|c|c|}
\hline \multirow{3}{*}{$\begin{array}{l}\text { Season } \\
\text { Month }\end{array}$} & \multicolumn{4}{|c|}{ Bubbler irrigation } & \multicolumn{4}{|c|}{ Surface irrigation } \\
\hline & \multicolumn{2}{|c|}{2011} & \multicolumn{2}{|c|}{2012} & \multicolumn{2}{|c|}{2011} & \multicolumn{2}{|c|}{2012} \\
\hline & $\begin{array}{c}\mathrm{m}^{3} / \text { fed./ } \\
\text { day }\end{array}$ & $\begin{array}{c}\mathrm{m}^{3} / \mathrm{fed} \\
. / \\
\text { month }\end{array}$ & $\begin{array}{c}\mathrm{m}^{3} / \text { fed. } \\
\text { / day }\end{array}$ & $\begin{array}{c}\mathrm{m}^{3} / \text { fed. } \\
/ \\
\text { month }\end{array}$ & $\begin{array}{c}\mathrm{m}^{3} / \text { fed. } \\
\text { / day }\end{array}$ & $\begin{array}{c}\mathrm{m}^{3} / \text { fed. } \\
/ \\
\text { month }\end{array}$ & $\begin{array}{l}\mathrm{m}^{3} / \text { fed. } \\
\text { / day }\end{array}$ & $\begin{array}{c}\mathrm{m}^{3} / \text { fed } \\
. / \\
\text { month }\end{array}$ \\
\hline January & 5.6 & 173 & 5.5 & 170 & - & - & - & - \\
\hline February & 6.9 & 192 & 6.5 & 188 & 8.2 & 230 & 6.9 & 200 \\
\hline March & 7.7 & 237 & 7.7 & 237 & 13.9 & 430 & 13.6 & 420 \\
\hline April & 11.4 & 342 & 11.2 & 336 & 17.7 & 530 & 17.7 & 530 \\
\hline May & 15.3 & 475 & 14.9 & 462 & 26.8 & 830 & 25.8 & 800 \\
\hline June & 18.1 & 544 & 15.6 & 469 & 46.0 & 1380 & 45.7 & 1370 \\
\hline July & 18.1 & 562 & 15.6 & 482 & 46.1 & 1430 & 46.1 & 1430 \\
\hline August & 17.6 & 545 & 15.6 & 483 & 42.9 & 1330 & 39.7 & 1230 \\
\hline September & 13.6 & 409 & 12.3 & 370 & 24.2 & 730 & 24.3 & 730 \\
\hline October & 12.1 & 374 & 10.3 & 319 & 13.2 & 410 & 12.9 & 400 \\
\hline November & 8.9 & 267 & 7.8 & 235 & 6.0 & 180 & 6.7 & 200 \\
\hline December & 5.9 & 182 & 5.2 & 162 & - & - & - & - \\
\hline Seasonal ( $\mathrm{m}^{3} /$ fed.) & - & 4302 & - & 3913 & - & 7480 & - & 7310 \\
\hline
\end{tabular}

\section{A-2. Monthly applied irrigation water.}

Monthly applied irrigation water values for orange trees were obtained from daily water use multiplied by the number of days in one month. It began to raise during March then, ET value gradually increased to reach its maximum at early summer during June and July Fig. (1). This might be due to the increase in growth during summer months afterwards, the daily applied irrigation water, again, gradually decreased. Such pattern was attained by orange trees, regardless of factors studied. In this concern, during April and early May little growth will appear, but towards the end of October the trees slow down into steady progress. Similar results were obtained since water management practices resulted in maximum yield, and trees growth depending on crop load and yearly climatic change. There was an increase in transpiration and water uptake from summer to autumn followed by a decrease until spring. 

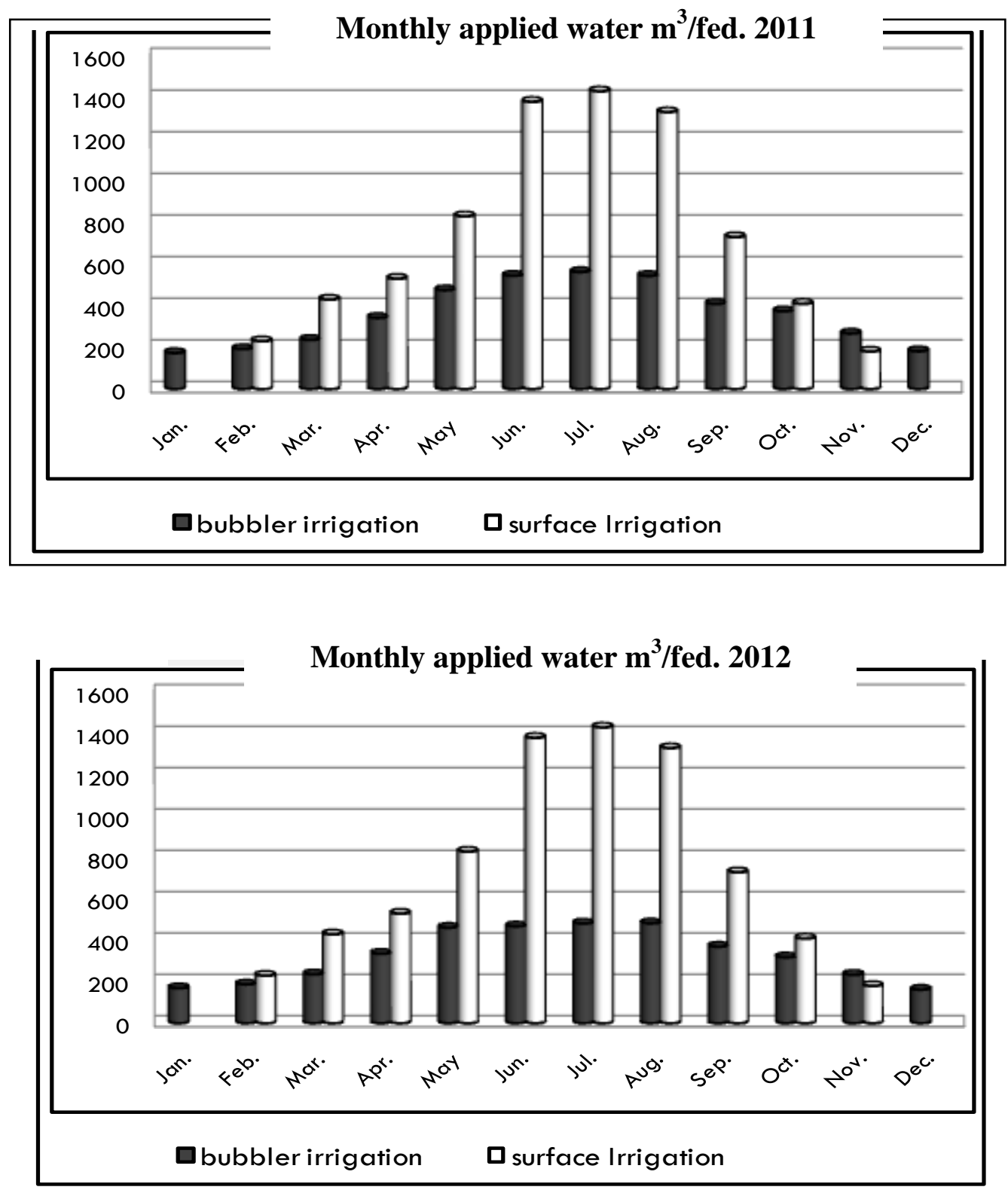

Fig. 1, Monthly applied irrigation water to orange trees by irrigation system in 2011 and 2012 growing seasons.

\section{A-3. Water utilization efficiency (W.U.E):}

Water utilization efficiency, is used to show the yield (kg.) per unit of water required in evapotranspiration. It appears from Fig. 2 that this trait was markedly profitable under bubbler irrigation as it reached $\left(4.93\right.$ yield $\mathrm{kg} / \mathrm{m}^{3}$ ) water consumed, while it decreased in case of surface irrigation and reached $\left(2.0 \mathrm{~kg} / \mathrm{m}^{3}\right)$ water consumed, as average of two seasons of study, respectively. So, bubbler irrigation method could raise water utilization efficiency to $59.4 \%$ compared to surface method. 


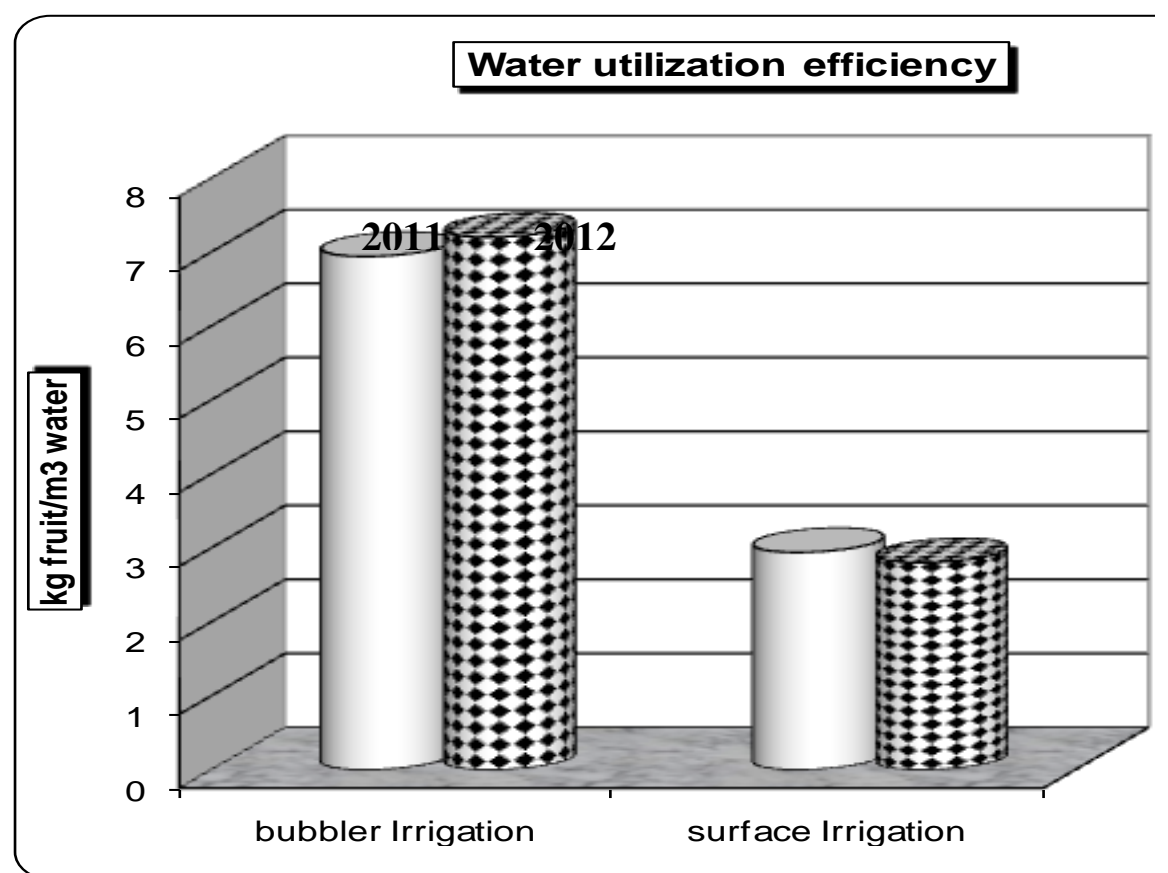

Fig. 2. Effect of bubbler irrigation and surface irrigation on water utilization efficiency (W.U.E) $\mathrm{kg} / \mathrm{m}^{3}$ of orange trees.

\section{B- Tree growth:}

\section{B-1. Vegetative growth measurements:}

Table (3) show the effect of irrigation methods on some vegetative growth parameters of Washington Navel orange trees as expressed by shoot length, number of leaves per shoot and leaf area during both 2011 and 2012 seasons.

Concerning the shoot length $(\mathrm{cm}$.), it is obvious that trees irrigated by bubbler irrigation system produced higher shoot length growth parameter (the longest shoots) in both seasons, compared with surface irrigation system. These differences were significant when compared to each other in the first and second seasons.

With respect to number of leaves/shoot, it is clear that, trees irrigated by surface irrigation system produced significantly the largest number of leaves per shoot and subsequently higher evapotranspiration rate.

Leaf area $\left(\mathrm{cm}^{2}\right)$ of Washington Navel orange trees, table (3) indicated that the existing differences between bubbler irrigation and surface systems were found to be significant in both seasons (2011 and 2012) under study. The highest increase in leaf area $\left(\mathrm{cm}^{2}\right)$ was recorded in case of bubbler irrigation method.

Table 3. Shoot length, number of leaves/shoot and leaf area of Washington Navel orange trees in response to surface and bubbler irrigation system during 2011 and 2012 seasons.

\begin{tabular}{|c|c|c|c|c|c|c|}
\hline \multirow{2}{*}{ Irrigation system } & \multicolumn{2}{|l|}{ Shoot length $(\mathrm{cm})}$. & \multicolumn{2}{c|}{$\begin{array}{c}\text { No. of leaves/ } \\
\text { shoot }\end{array}$} & \multicolumn{2}{c|}{ Leaf area $\left(\mathrm{cm}^{2}\right)$} \\
\cline { 2 - 7 } & 2011 & 2012 & 2011 & 2012 & 2011 & 2012 \\
\hline Bubbler irrigation & $15.67 \mathrm{~A}$ & $15.33 \mathrm{~A}$ & $18.00 \mathrm{~B}$ & $16.00 \mathrm{~B}$ & $25.37 \mathrm{~A}$ & $22.85 \mathrm{~A}$ \\
\hline Surface irrigation & $14.0 \mathrm{~B}$ & $13.33 \mathrm{~B}$ & $22.0 \mathrm{~A}$ & $20.67 \mathrm{~A}$ & $21.72 \mathrm{~B}$ & $20.47 \mathrm{~B}$ \\
\hline
\end{tabular}


It is clear that transition from surface irrigation to bubbler irrigation system exerts positive effects on vegetative growth of orange trees. Plants spend most of their energies while taking water from the soil by their roots. Because, the irrigation interval is long during surface irrigation and soil moisture water decreases continuously after irrigation, roots of trees spend most of their energies in water absorption and spend less energy for growth and development. In bubbler irrigation, as the soil is more humid due to frequent irrigation intervals the trees do not spend much energy while taking water from the soil. They spend most of their energies for growth, development, productivity and fruit quality. Therefore, the vegetative growth of bubbler irrigation treatments was positively influenced. Shoot length, leaf area and number of shoot increased in case of bubbler compared to those of surface irrigation. The reason of the increase in these values is the positive impact on vegetative growth. Similarly, Hilgeman and Sharo (1970), Abdel-Missih et al., (1977) and Ali and Gobran (2002) on Washington Navel orange pointed out that fruit trees which had been irrigated for many years with surface irrigation systems did not indicate any reduction in vegetative growth after switching to bubbler irrigation, and bubbler irrigation had a positive effect on vegetative growth. Some researchers reported that different irrigation schedules on plum and pistachio trees and different irrigation systems on lemon trees had no effects on vegetative growth.

\section{B-2. Length and number of roots:}

Data obtained in tables ( $4 \& 5$ ) show that roots length with different diameters either at horizontal or vertical directions were significantly increased using bubbler irrigation system than surface system. Also, it was observed that, roots were concentrated around the trunk and around the bubbler $(100 \mathrm{~cm}$ from tree trunk) especially at $0-30 \mathrm{~cm}$ in depth. The majority of root system is fine roots $(<2 \mathrm{~mm})$ while $>2 \mathrm{~mm}$ roots extended only to $(100 \mathrm{~cm})$ from the tree trunk.

\section{B-3. Dry weight of roots:}

Furthermore, data in Table (6) show obviously the root dry weight of Washington Navel orange trees at $(100,200$ and $300 \mathrm{~cm})$ distances from the tree trunk as well as at $(0-30,30-60 \& 60-90 \mathrm{~cm})$ depths from the soil surface as affected by both bubbler and surface irrigation systems. The data indicate that, root dry weight significantly and gradually reduced as irrigation water decreased by increasing the distance from the tree trunk i.e., ( 1.31 to 0.97 to 0.46 (in case of $<2 \mathrm{~mm}$ thick roots) and $0.86,2.76$ to $1.99 \mathrm{~g}$. for root of $>2 \mathrm{~mm}$ ). Whereas, the reduction of root dry weight was pronounced by increasing the depth of the soil surface $(1.21,1.39$ to 0.14 for roots $<2 \mathrm{~mm}$ ) and $1.62,3.91$ and $0.08 \mathrm{~g}$. for roots $>2 \mathrm{~mm}$ ).

Root distribution depends upon the volume of wetted soil, which was related to soil hydraulic conductivity as well as the rate and duration of water application (Levin et al., 1980). Reducing the size of the root zone decreased the size of root system and caused a decrease in water consumption. Meanwhile, root length was significantly and negatively 
affected by water stress and the highest decrease occurred when the available soil water decreased from 40 to $20 \%$ (Fathi, 1999b and Ibrahim, 2001).

These results support the phenomenon that water stress reduced root distribution and soil dryness significantly reduced root dry matter production. The present results also showed that the average dry weight of large roots ( $>2 \mathrm{~mm}$ ) was considerably higher than fine roots $(<2 \mathrm{~mm}$ ) specially at 0-30 and 30-60 cm depth at 200 $\mathrm{cm}$ from tree trunk. Fathi (1999 b) stated that, dry weight significantly reduced at low level irrigation whereas more than $90 \%$ of roots were $80 \mathrm{~cm}$ from the trunk. He also indicated that water stress decreased root densities at shallow soil depths.

Table 4. Root length $(\mathrm{cm}$.) of Washington Navel orange trees in response to both surface irrigation system and bubbler irrigation system during 2012 season.

\begin{tabular}{|c|c|c|c|c|c|c|c|c|c|}
\hline \multirow{3}{*}{$\begin{array}{l}\text { Irrigation } \\
\text { system (A) }\end{array}$} & \multirow{3}{*}{$\begin{array}{c}\text { Distances } \\
\text { (B) }\end{array}$} & \multicolumn{4}{|c|}{ Roots thicker less than $2 \mathrm{~mm}(\mathrm{C}$ ) } & \multicolumn{4}{|c|}{ Roots thicker than $2 \mathrm{~mm}$ ( C ) } \\
\hline & & \multicolumn{4}{|c|}{ (root depths $\mathrm{cm}$ ) } & \multicolumn{4}{|c|}{ (root depths $\mathrm{cm}$ ) } \\
\hline & & $0-30$ & $30-60$ & $60-90$ & $\begin{array}{c}\text { Average } \\
(A \times B)\end{array}$ & $0-30$ & $30-60$ & $60-90$ & $\begin{array}{c}\text { Average } \\
(A \times B)\end{array}$ \\
\hline \multirow{3}{*}{ Bubbler } & $100 \mathrm{~cm}$ & $881.8 \mathrm{a}$ & $317.7 e$ & $20.17 \mathrm{~h}$ & $406.6 \mathrm{~A}$ & $53.33 a$ & $32.8 \mathrm{bc}$ & $0.0 \mathrm{~g}$ & $28.72 \mathrm{~A}$ \\
\hline & $200 \mathrm{~cm}$ & $433.2 \mathrm{c}$ & $463.5 c$ & 49.83gh & $315.5 B$ & $9.00 \mathrm{fg}$ & $37.2 \mathrm{fb}$ & $9.3 \mathrm{fg}$ & $18.50 \mathrm{BC}$ \\
\hline & $300 \mathrm{~cm}$ & $149.9 f$ & $322.4 d$ & $40.5 \mathrm{gh}$ & 170.9D & $3.0 \mathrm{fg}$ & $28.0 \mathrm{~b}-\mathrm{e}$ & $0.0 \mathrm{~g}$ & $10.33 \mathrm{C}$ \\
\hline \multicolumn{2}{|c|}{ Average $(\mathrm{A} \times \mathrm{C})$} & $488.3 \mathrm{~A}$ & $367.8 \mathrm{~B}$ & 36.83D & $\begin{array}{l}\text { Ave. A } \\
297.7 \mathrm{~A}\end{array}$ & 21.78B & $32.7 \mathrm{~A}$ & 3.11CD & $\begin{array}{l}\text { Ave. A } \\
19.19 \text { A }\end{array}$ \\
\hline \multirow{3}{*}{ Surface } & $100 \mathrm{~cm}$ & $596.0 \mathrm{~b}$ & $170.0 f$ & $17.17 \mathrm{~h}$ & $261.0 \mathrm{C}$ & $57.33 a$ & $12.17 \mathrm{~d}-\mathrm{g}$ & $4.33 \mathrm{fg}$ & $24.1 \mathrm{AB}$ \\
\hline & $200 \mathrm{~cm}$ & $384.4 d$ & $76.67 \mathrm{~g}$ & $17.33 \mathrm{~h}$ & 159.5D & $28.67 b-d$ & $11.67 \mathrm{e}-\mathrm{g}$ & $1.0 \mathrm{~g}$ & $13.78 \mathrm{C}$ \\
\hline & $300 \mathrm{~cm}$ & $170.1 f$ & $85.77 \mathrm{~g}$ & $10.00 \mathrm{~h}$ & $88.61 \mathrm{E}$ & $20.00 c-f$ & $11.0 \mathrm{fg}$ & $0.0 \mathrm{~g}$ & $10.33 C$ \\
\hline \multicolumn{2}{|c|}{ Average $(A \times C)$} & $383.5 B$ & $110.8 \mathrm{C}$ & $14.83 \mathrm{D}$ & $\begin{array}{l}\text { Ave. A } \\
\text { 169.7B }\end{array}$ & $35.33 \mathrm{~A}$ & $11.61 \mathrm{C}$ & $1.78 \mathrm{D}$ & $\begin{array}{l}\text { Ave. A } \\
16.24 \mathrm{~A}\end{array}$ \\
\hline Average & $100 \mathrm{~cm}$ & $738.9 A$ & $243.8 C$ & 18.7E & $\begin{array}{l}\text { Ave. B } \\
333.8 \mathrm{~A}\end{array}$ & $55.33 \mathrm{~A}$ & $22.50 \mathrm{BC}$ & $2.17 \mathrm{C}$ & $\begin{array}{l}\text { Ave. B } \\
26.7 D\end{array}$ \\
\hline \multirow[t]{2}{*}{$(B \times C)$} & $200 \mathrm{~cm}$ & 408.8B & $270.1 C$ & $33.6 \mathrm{E}$ & $237.5 B$ & $18.83 B C$ & $24.42 B$ & 5.17D & 16.1D \\
\hline & $300 \mathrm{~cm}$ & 160.0D & 204.1CD & $25.3 \mathrm{E}$ & $129.8 \mathrm{C}$ & $11.50 \mathrm{CD}$ & 19.50BC & $0.00 \mathrm{D}$ & 10.3D \\
\hline \multicolumn{2}{|c|}{ Average (C) } & $435.9 \mathrm{~A}$ & 239..3B & $25.83 C$ & - & $28.56 \mathrm{~A}$ & $22.14 \mathrm{~B}$ & $2.44 \mathrm{C}$ & - \\
\hline
\end{tabular}

Values having the same letter (s) within the same column are not statistically significant.

Table 5. Number of roots of Washington Navel orange trees in response to both surface irrigation system and bubbler irrigation system during 2012 season.

\begin{tabular}{|c|c|c|c|c|c|c|c|c|c|}
\hline \multirow{3}{*}{$\begin{array}{c}\text { Irrigation } \\
\text { system (A) }\end{array}$} & \multirow{3}{*}{$\begin{array}{c}\text { Distances } \\
\text { (B) }\end{array}$} & \multicolumn{4}{|c|}{ Roots thicker less than $2 \mathrm{~mm}(\mathrm{C})$} & \multicolumn{4}{|c|}{ Roots thicker than $2 \mathrm{~mm}(\mathrm{C})$} \\
\hline & & \multicolumn{4}{|c|}{ (root depths $\mathrm{cm})$} & \multicolumn{4}{|c|}{ (root depths $\mathrm{cm}$ ) } \\
\hline & & $0-30$ & $30-60$ & $60-90$ & $\begin{array}{c}\text { Average } \\
(\mathrm{A} \times \mathrm{B})\end{array}$ & $0-30$ & $30-60$ & $60-90$ & $\begin{array}{c}\text { Average } \\
(\mathrm{A} \times \mathrm{B})\end{array}$ \\
\hline \multirow{3}{*}{ Bubbler } & $100 \mathrm{~cm}$ & $593.0 \mathrm{a}$ & $144.3 \mathrm{c}$ & $9.0 \mathrm{~d}$ & $248.8 \mathrm{~A}$ & $9.00 a$ & $5.67 \mathrm{bc}$ & $0.0 f$ & $4.89 \mathrm{~A}$ \\
\hline & $200 \mathrm{~cm}$ & $392.7 \mathrm{~b}$ & $360.0 \mathrm{~b}$ & $27.0 \mathrm{~cd}$ & $259.9 \mathrm{~A}$ & $3.00 \mathrm{c}-\mathrm{f}$ & $5.00 \mathrm{~cd}$ & $2.00 d-f$ & $3.33 \mathrm{~A}-\mathrm{C}$ \\
\hline & $300 \mathrm{~cm}$ & 74.7cd & $150.0 \mathrm{c}$ & $14.0 \mathrm{~d}$ & $79.6 \mathrm{BC}$ & $2.00 \mathrm{~d}-\mathrm{f}$ & $4.67 \mathrm{~cd}$ & $0.00 f$ & $2.22 \mathrm{C}$ \\
\hline \multicolumn{2}{|c|}{ Average $(\mathrm{A} \times \mathrm{C})$} & $353.4 \mathrm{~A}$ & 218.1B & $16.7 \mathrm{C}$ & $\begin{array}{l}\text { Ave. A } \\
196.1 \mathrm{~A}\end{array}$ & 4.67A & $5.11 \mathrm{~A}$ & $0.67 \mathrm{~B}$ & $\begin{array}{c}\text { Ave. A } \\
3.48 \mathrm{~A}\end{array}$ \\
\hline \multirow{3}{*}{ Surface } & $100 \mathrm{~cm}$ & $535.0 a$ & $93.3 \mathrm{~cd}$ & $9.0 \mathrm{~d}$ & $212.4 \mathrm{~A}$ & $8.00 \mathrm{ab}$ & $4.33 \mathrm{~cd}$ & $1.00 \mathrm{ef}$ & $4.44 A B$ \\
\hline & $200 \mathrm{~cm}$ & $300.0 \mathrm{~b}$ & $47.0 \mathrm{~cd}$ & $13.0 \mathrm{~d}$ & $120.0 \mathrm{~B}$ & $3.33 c-e$ & $4.67 \mathrm{~cd}$ & $1.0 \mathrm{ef}$ & $3.00 \mathrm{BC}$ \\
\hline & $300 \mathrm{~cm}$ & 95.7cd & $44.0 \mathrm{~cd}$ & $8.0 \mathrm{~d}$ & $49.2 \mathrm{C}$ & $4.0 c-e$ & $3.33 c-e$ & $0.0 f$ & $2.44 \mathrm{C}$ \\
\hline \multicolumn{2}{|c|}{ Average $(A \times C)$} & $310.2 \mathrm{~A}$ & $61.4 \mathrm{C}$ & $10.0 \mathrm{C}$ & $\begin{array}{l}\text { Ave. A } \\
127.2 B\end{array}$ & $5.11 \mathrm{~A}$ & $4.11 \mathrm{~A}$ & $0.67 \mathrm{~B}$ & $\begin{array}{c}\text { Ave. A } \\
3.30 \mathrm{~A}\end{array}$ \\
\hline \multirow{3}{*}{$\begin{array}{c}\text { Average } \\
(B \times C)\end{array}$} & $100 \mathrm{~cm}$ & 564.A & 118.8D & $9.0 \mathrm{~F}$ & $\begin{array}{l}\text { Ave. B } \\
230.6 \mathrm{~A}\end{array}$ & $8.50 \mathrm{~A}$ & $5.00 \mathrm{~B}$ & $0.50 \mathrm{D}$ & $\begin{array}{c}\text { Ave. B } \\
4.67 A\end{array}$ \\
\hline & $200 \mathrm{~cm}$ & $346.3 \mathrm{~B}$ & $203.5 \mathrm{C}$ & 20.0EF & $189.9 \mathrm{~A}$ & $3.17 \mathrm{BC}$ & $4.83 \mathrm{~B}$ & $1.50 \mathrm{CD}$ & 3.17B \\
\hline & $300 \mathrm{~cm}$ & 85.2D-F & $97.0 \mathrm{DE}$ & $11.0 \mathrm{~F}$ & $64.4 \mathrm{~B}$ & $3.00 \mathrm{BC}$ & $4.00 \mathrm{~B}$ & $0.00 \mathrm{D}$ & $2.33 \mathrm{~B}$ \\
\hline \multicolumn{2}{|c|}{ Average (C) } & $331.8 \mathrm{~A}$ & 139.8B & $13.3 \mathrm{C}$ & - & $4.89 \mathrm{~A}$ & $4.61 \mathrm{~A}$ & $0.67 \mathrm{~B}$ & - \\
\hline
\end{tabular}

Values having the same letter (s) within the same column are not statistically significant. 
Table 6. Root dry weight (g.) of Washington Navel orange trees in response to both surface irrigation system and bubbler irrigation system during 2012 season.

\begin{tabular}{|c|c|c|c|c|c|c|c|c|c|}
\hline \multirow{3}{*}{$\begin{array}{l}\text { Irrigation } \\
\text { system (A) }\end{array}$} & \multirow{3}{*}{$\begin{array}{c}\text { Distances } \\
\text { (B) }\end{array}$} & \multicolumn{4}{|c|}{ Roots thicker less than $2 \mathrm{~mm}(\mathrm{C})$} & \multicolumn{4}{|c|}{ Roots thicker than $2 \mathrm{~mm}(\mathrm{C})$} \\
\hline & & \multicolumn{4}{|c|}{ (root depths $\mathrm{cm}$ ) } & \multicolumn{4}{|c|}{ (root depths $\mathrm{cm}$ ) } \\
\hline & & $0-30$ & $30-60$ & $60-90$ & $\begin{array}{l}\text { Average } \\
(\mathrm{A} \times \mathrm{B})\end{array}$ & $0-30$ & $30-60$ & $60-90$ & $\begin{array}{r}\text { Average } \\
(\mathrm{A} \times \mathrm{B})\end{array}$ \\
\hline \multirow{3}{*}{ Bubbler } & $100 \mathrm{~cm}$ & $1.87 \mathrm{bc}$ & $3.86 a$ & $0.07 \mathrm{f}$ & $1.93 \mathrm{~A}$ & $0.74 c$ & $2.00 \mathrm{c}$ & $0.0 \mathrm{c}$ & $0.91 \mathrm{C}$ \\
\hline & $200 \mathrm{~cm}$ & $1.29 \mathrm{~b}-\mathrm{e}$ & $2.19 \mathrm{~b}$ & $0.35 \mathrm{ef}$ & $1.28 \mathrm{~B}$ & $0.17 c$ & $13.23 a$ & $0.29 c$ & $4.56 \mathrm{~A}$ \\
\hline & $300 \mathrm{~cm}$ & $0.60 \mathrm{~d}-\mathrm{f}$ & $1.08 \mathrm{c}-\mathrm{f}$ & $0.15 \mathrm{f}$ & $0.61 \mathrm{C}$ & $0.10 c$ & $6.38 \mathrm{~b}$ & $0.0 \mathrm{c}$ & $2.16 \mathrm{~B}$ \\
\hline \multicolumn{2}{|c|}{ Average $(A \times C)$} & $1.26 \mathrm{~B}$ & $2.37 \mathrm{~A}$ & $0.19 \mathrm{C}$ & $\begin{array}{l}\text { Ave. A } \\
1.27 \mathrm{~A}\end{array}$ & $0.33 \mathrm{C}$ & $7.20 \mathrm{~A}$ & $0.10 \mathrm{C}$ & $\begin{array}{l}\text { Ave. A } \\
2.55 \mathrm{~A}\end{array}$ \\
\hline \multirow{3}{*}{ Surface } & $100 \mathrm{~cm}$ & $1.50 \mathrm{~b}-\mathrm{d}$ & $0.39 d-f$ & $0.14 \mathrm{f}$ & $0.68 \mathrm{C}$ & $1.61 \mathrm{c}$ & $0.66 \mathrm{c}$ & $0.13 c$ & $0.80 \mathrm{C}$ \\
\hline & $200 \mathrm{~cm}$ & $1.49 \mathrm{~b}-\mathrm{d}$ & $0.44 d-f$ & $0.07 f$ & $0.67 \mathrm{C}$ & $2.12 c$ & $0.70 c$ & $0.03 c$ & $0.95 \mathrm{C}$ \\
\hline & $300 \mathrm{~cm}$ & $0.49 \mathrm{~d}-\mathrm{f}$ & $0.39 d-f$ & $0.07 f$ & $0.31 \mathrm{C}$ & $4.99 \mathrm{~b}$ & $0.48 \mathrm{c}$ & $0.00 c$ & $1.83 \mathrm{BC}$ \\
\hline \multicolumn{2}{|c|}{ Average $(A \times C)$} & $1.16 \mathrm{~B}$ & $0.41 \mathrm{C}$ & $0.09 \mathrm{C}$ & $\begin{array}{c}\text { Ave. A } \\
0.55 \mathrm{~B} \\
\end{array}$ & $2.91 \mathrm{~B}$ & $0.61 \mathrm{C}$ & $0.06 \mathrm{C}$ & $\begin{array}{c}\text { Ave. A } \\
1.19 \mathrm{~B} \\
\end{array}$ \\
\hline \multirow{3}{*}{$\begin{array}{c}\text { Average } \\
(B \times C)\end{array}$} & $100 \mathrm{~cm}$ & $1.69 \mathrm{AB}$ & $2.13 \mathrm{~A}$ & $0.11 \mathrm{D}$ & $\begin{array}{l}\text { Ave. B } \\
1.31 \mathrm{~A} \\
\end{array}$ & $1.18 \mathrm{CD}$ & $1.33 \mathrm{CD}$ & $0.07 \mathrm{D}$ & $\begin{array}{l}\text { Ave. B } \\
0.86 \mathrm{~B}\end{array}$ \\
\hline & $200 \mathrm{~cm}$ & $1.39 \mathrm{BC}$ & $1.31 \mathrm{BC}$ & $0.21 \mathrm{D}$ & $0.97 \mathrm{~A}$ & $1.14 \mathrm{CD}$ & $6.97 \mathrm{~A}$ & $0.16 \mathrm{D}$ & $2.76 \mathrm{~A}$ \\
\hline & $300 \mathrm{~cm}$ & $0.55 \mathrm{D}$ & $0.73 C D$ & $0.11 \mathrm{D}$ & $0.46 \mathrm{~B}$ & $2.55 \mathrm{BC}$ & $3.43 \mathrm{~B}$ & $0.00 \mathrm{D}$ & $1.99 \mathrm{~A}$ \\
\hline \multicolumn{2}{|c|}{ Average (C) } & $1.21 \mathrm{~A}$ & $1.39 \mathrm{~A}$ & $0.14 \mathrm{~B}$ & - & $1.62 \mathrm{~B}$ & $3.91 \mathrm{~A}$ & $0.08 \mathrm{C}$ & - \\
\hline
\end{tabular}

Values having the same letter (s) within the same column are not statistically significant.

\section{C- Fruiting parameters.}

\section{C-1. Yield $\mathrm{kg} /$ tree and number of fruits per tree.}

Considering yield as $\mathrm{kg}$. per tree, it is interesting to notice from data in Table (7) that, trees irrigated with bubbler irrigation system, yielded greater amounts of fruit crop (177.8 and $167.7 \mathrm{~kg}$ tree) than those of trees irrigated by surface irrigation system (131.0 and $121.9 \mathrm{~kg} /$ tree) for the two seasons, respectively.

With respect to tree yield as calculated by number of fruits per tree, it is quite clear from data (Table, 7) that, a significant increase was tree noticed in fruit number per tree irrigated with bubbler irrigation system (554.3 and 650.0) than the analogous ones of trees irrigated by surface irrigation system ( 450.0 and 446.7 tree). Such trend was true for both 2011 and 2012 seasons.

\section{C-2. Fruit weight and volume.}

Concerning the average fruit weight (g.) and fruit size $\left(\mathrm{cm}^{3}\right)$ as affected by both irrigation systems under study, data in table (7) indicated that, Washington Navel orange trees irrigated by bubbler irrigation system produced fruits of heaviest weight and greatest size. Such trend was true during 2011 and 2012 seasons of study. 
Table 7. Yield $\mathrm{kg} /$ tree, number of fruits/tree, fruit weight (g.) and fruit size $\left(\mathrm{cm}^{3}\right)$ of Washington Navel orange trees in response to both surface and bubbler irrigation systems during 2011 and 2012 seasons.

\begin{tabular}{|c|c|c|c|c|c|c|c|c|}
\hline \multirow{2}{*}{ Irrigation system } & \multicolumn{2}{|c|}{ Yield $(\mathrm{kg} /$ tree $)$} & \multicolumn{2}{c|}{ No. of fruits/tree } & \multicolumn{2}{c|}{ Fruit weight $(\mathrm{g})}$. & \multicolumn{2}{c|}{ Fruit size $\left(\mathrm{cm}^{3}\right)$} \\
\cline { 2 - 9 } & 2011 & 2012 & 2011 & 2012 & 2011 & 2012 & 2011 & 2012 \\
\hline Bubbler irrigation & $177.8 \mathrm{~A}$ & $167.7 \mathrm{~A}$ & $554.3 \mathrm{~A}$ & $650.0 \mathrm{~A}$ & $318.2 \mathrm{~A}$ & $257.4 \mathrm{~A}$ & $372.8 \mathrm{~A}$ & $272.8 \mathrm{~A}$ \\
\hline Surface irrigation & $131.0 \mathrm{~B}$ & $121.9 \mathrm{~B}$ & $450.0 \mathrm{~B}$ & $446.7 \mathrm{~B}$ & $290.9 \mathrm{~A}$ & $235.0 \mathrm{~A}$ & $342.5 \mathrm{~A}$ & $240.4 \mathrm{~A}$ \\
\hline
\end{tabular}

\section{C-3. Fruit polar and equatorial diameter:}

With regard to both polar and equatorial diameter $(\mathrm{cm}$.$) of Washington Navel$ orange trees, it is quite evident that, trees irrigated with surface system induced large fruit diameter and length in first season compared to those irrigated by bubbler irrigation method however, the opposite trend exists in $2^{\text {nd }}$ season. Meanwhile, differences between the two irrigated methods under study were insignificant. Peel thickness has such trend but with significant differences in $2^{\text {nd }}$ season.

Table 8. Polar diameter $(\mathrm{cm}$.$) , equatorial diameter (\mathrm{cm}$.$) and peel thickness, of$ Washington Navel orange trees in response to both surface and bubbler irrigation system during 2011 and 2012 seasons.

\begin{tabular}{|l|c|c|c|c|c|c|}
\hline \multirow{2}{*}{ Irrigation system } & \multicolumn{2}{|c|}{$\begin{array}{c}\text { Polar diameter } \\
(\mathrm{cm} .)\end{array}$} & $\begin{array}{c}\text { Equatorial diameter } \\
(\mathrm{cm} .)\end{array}$ & \multicolumn{2}{c|}{$\begin{array}{c}\text { Peel thickness } \\
(\mathrm{mm})\end{array}$} \\
\cline { 2 - 7 } & 2011 & 2012 & 2011 & 2012 & 2011 & 2012 \\
\hline Bubbler irrigation & $8.17 \mathrm{~A}$ & $7.95 \mathrm{~A}$ & $8.65 \mathrm{~A}$ & $8.44 \mathrm{~A}$ & $4.27 \mathrm{~A}$ & $4.70 \mathrm{~A}$ \\
\hline Surface irrigation & $8.19 \mathrm{~A}$ & $7.63 \mathrm{~A}$ & $9.0 \mathrm{~A}$ & $7.80 \mathrm{~A}$ & $4.37 \mathrm{~A}$ & $3.27 \mathrm{~B}$ \\
\hline
\end{tabular}

Positive effects of transition from surface to bubbler irrigation system were identified on fruit quality. Fruit diameter, length and fruit weight were lower in surface than bubbler irrigation treatments. In view of peel thickness, Hilgeman and Sharo (1970) on Valencia orange trees found that low supply water increased peel thickness.

\section{C-3. Fruit chemical properties:}

\section{C-3-1. Fruit juice total soluble solids:}

Data presented in table (9) show obviously the effect of both bubbler and surface irrigation systems on fruit juice total soluble solids during 2011 and 2012 seasons of study. Surface irrigation gave the highest value of TSS \% than the other irrigation system (bubbler).

\section{C-3-2. Fruit juice total acidity:}

Table (9) cleared that bubbler irrigation system effectively reduced the percentage of juice acidity to 0.793 and $0.800 \%$ in the two studied seasons, respectively. However, this result was confirmed statistically in the first season. 


\section{C-3-3. Ascorbic acid content (vitamin C).}

It is quite evident from table (9) that, the highest value of ascorbic acid content exists in case of surface irrigation compared with the method which induced the least significant value in this respect.

Fruit quality results under irrigation regimes are in agreement with the findings of Hilgeman and Sharo (1970) on orange and Ali and Gobran (2002) on Washington Navel orange, they mentioned that higher applied water decreased TSS, total acidity and ascorbic acid contents. In this concern, Naguib (1978) mentioned that prolonged drought period increased total soluble solid in the juice of Balady orange fruit, this may be attributed to moisture content of fruit in the unirrigated trees for long period so the TSS content was relatively high.

Table 9. Total soluble solids (\%), total acidity, TSS/acid ratio and ascorbic acid content of Washington Navel orange trees in response to both surface and bubbler irrigation system during 2011 and 2012 seasons.

\begin{tabular}{|c|c|c|c|c|c|c|c|c|}
\hline \multirow{2}{*}{ Irrigation system } & \multicolumn{2}{|c|}{ TSS \% } & \multicolumn{2}{c|}{ Acidity \% } & \multicolumn{2}{c|}{ TSS/acid ratio } & \multicolumn{2}{c|}{$\begin{array}{c}\text { Ascorbic acid } \\
\text { (mg/100 ml) }\end{array}$} \\
\cline { 2 - 9 } & 2011 & 2012 & 2011 & 2012 & 2011 & 2012 & 2011 & 2012 \\
\hline Bubbler irrigation & $11.57 \mathrm{~B}$ & $11.50 \mathrm{~B}$ & $0.793 \mathrm{~B}$ & $0.800 \mathrm{~A}$ & $14.59 \mathrm{~A}$ & $14.38 \mathrm{~A}$ & $35.8 \mathrm{~B}$ & $32.59 \mathrm{~A}$ \\
\hline Surface irrigation & $12.46 \mathrm{~A}$ & $12.50 \mathrm{~A}$ & $0.833 \mathrm{~A}$ & $0.833 \mathrm{~A}$ & $14.96 \mathrm{~A}$ & $15.01 \mathrm{~A}$ & $36.3 \mathrm{~A}$ & $33.12 \mathrm{~A}$ \\
\hline
\end{tabular}

\section{D- Leaf mineral composition (leaf macronutrients content):}

Considering the leaf content of some macro elements, i.e., ( $N, \mathrm{P}$ and $\mathrm{K}$ ) of Washington Navel orange trees under the two irrigation system (surface and bubbler systems), data tabulated in table (10) show obviously that variations due to the effect of irrigation systems under study were so little to reach level of significance. It could be noticed that, the absence of significance in the response of $(N, P \& K)$ leaf content to both surface irrigation system and bubbler irrigation system was detected during both the first and second seasons of study.

Table 10. Nitrogen, phosphorus and potassium (\%) of Washington Navel orange trees in response to both surface and bubbler irrigation system during 2011 and 2012 seasons.

\begin{tabular}{|c|c|c|c|c|c|c|}
\hline \multirow{2}{*}{ Irrigation system } & \multicolumn{2}{|c|}{ Nitrogen (\%) } & \multicolumn{2}{c|}{ Phosphorus \% } & \multicolumn{2}{c|}{ Potassium (\%) } \\
\cline { 2 - 7 } & 2011 & 2012 & 2011 & 2012 & 2011 & 2012 \\
\hline Bubbler irrigation & $2.060 \mathrm{~A}$ & $2.05 \mathrm{~A}$ & $0.163 \mathrm{~A}$ & $0.152 \mathrm{~A}$ & $1.40 \mathrm{~A}$ & $1.35 \mathrm{~A}$ \\
\hline Surface irrigation & $2.045 \mathrm{~A}$ & $2.03 \mathrm{~A}$ & $0.160 \mathrm{~A}$ & $0.150 \mathrm{~A}$ & $1.43 \mathrm{~A}$ & $1.37 \mathrm{~A}$ \\
\hline
\end{tabular}

The obtained results are in conformity with that previously mentioned by Ali and Gobran (2002) on Washington Navel orange, they mentioned that water stress 
reduced the leaf nitrogen content, while Ibrahim (2001) found that water logged citrus seedlings recorded the lowest nitrogen percentage than both normal and stressed seedlings.

\section{GENERAL DISCUSSION}

The present results cleared that water utilization efficiency under bubbler irrigation system (4.93 kg fruits $/ \mathrm{m}^{3}$ irrigation water) is significantly higher than under surface system $\left(2.0 \mathrm{~kg} / \mathrm{m}^{3}\right)$. Also, vegetative growth (as shoot length and leaf area), fruiting parameters (as fruit yield and No. of fruits/tree), fruit juice acidity as well as root system distribution (as root length, number of roots and root dry weight) were positively better under bubbler system than surface one. Hence, bubbler irrigation system has all these benefits and saves about half irrigation quantities (Table 2).

However, when the controllable variables are optimized in order to avoid any occurrence of moisture stress during the growing season, many crops show a pronounced increase in yield. The desired effect can be produced by optimizing the quantity and increasing the frequency of irrigation, taking care of avoiding wetting soil excessively. This optimization is difficult to achieve by the traditional surface irrigation methods. The advantages of newer irrigation systems have made it possible to establish and maintain soil moisture conditions at a more nearly optimal level. These new systems are capable of delivering water in controllable small quantities as after and as long as needed (Hillel, 1987).

The old average trees ( 35 years) were surface irrigated since farm initiation, then subjected to bubbler and surface irrigation systems for comparison in this study. The data concerned with the root system (Tables 4 and 5) reveal that applying bubbler system resulted in concentrating the effective roots $<2 \mathrm{~mm}$ diameter in length and number in the wetted zone (100 $\mathrm{cm}$ from the trunk). In the same time, significant increase in orange yield and fruit characteristics were recorded in case of bubbler system also.

These results should be highly considered by decision makers in case of converting the surface irrigation system used in orchards of the old lands, which will result in saving irrigation water without affecting orange production.

Hussein (1998) on apple and Fathi (1999 ( $a$ and b) on pear revealed that drip irrigation at $80 \%$ F.C. gave the best results and increased yield by about $20 \%$. Also, Hussein and Eid (2013) recommended plum growers on clayey loamy soil to change flood irrigation system to drip system to save $50.1 \%$ of irrigation water with better growth, fruit yield, fruit quality, improved water use efficiency and reduce weed competition. 
In brief, it could be concluded and recommended for Washington Navel orange trees grown on clayey loamy soil to change the existing surface irrigation to bubbler system for saving irrigation water to about one half with better fruit yield, quality, longer root system and increasing water utilization efficiency to $59.6 \%$.

\section{REFERENCES}

1. Abdel-Missih, M. N., M.A. El- Nokrashy and G. Elham. 1977. Effect of different soil moisture levels on growth, yield and quality of Washington Navel orange. Agric. Res. Rev. Cairo. 55 No. 3:47-57.

2. Ali. M. A. and Y. N. Gobran. 2002. Effect of soil moisture regimes and potassium application on growth, yield, fruit quality of Washington Navel orange trees. Annals of Agric Sc., Moshtoher, Val.40 (3): 1669-1697.

3. A.O.A.C. 1990. Association of Official Agricultural chemists. Official Methods of Analysis. $12^{\text {th }}$ ed. Washington . D.C.

4. Brown, L. R. 1999. Feeding Nine Billions. In L. Storke (Ed. State of the world (1999). Norton and New York p.230.

5. Cahoon, G. A., E. S. Morton, W. W. Jane and M. J. Garber. 1959. Effect of various types of nitrogen fertilization on root density and distribution as related to water infiltration and fruit yield of "Washington Navel" orange in a long term fertilizer experiment. Proc. Amer. Soc. Hort. Sci., 74: 289-299.

6. Doorenbos J. and W.D. Pruitt. 1984. "Guidelines for Predicting Crop Water requirements, Irr. and Drainage Paper" $24,2^{\text {nd }}$ ed. FAO Roma, Italy.

7. Duncan, D. B. 1955. Multiple range and multiple F. tests. Biometrics, 11: 1-42.

8. Evenhuis, B. 1978. Simplified methods for foliar. Analysis, Koninklijk Institaut voorde tropen, Amsterdam, pp. 1-170.

9. Fathi, M. A. 1999 a. Drip irrigation efficiency for pear trees. A: Yield, fruit properties and vegetative growth. J. Agric. Sci., Mansoura Univ., 24 (6): 30213034.

10. Fathi, M. A. 1999 b. Drip irrigation efficiency for pear trees. B- Root system growth and distribution. J. Agric. Sci., Mansoura Univ., 24 (6): 3035-3049.

11. Ford, H. W. 1962. Thickness of sub soil organic layer in relation to tree size and root distribution of citrus. J. Amer. Soc. Hort. Sci., 82: 177-179. 
12. Hillel, D. 1987. The efficient use of water in irrigation. Principles and Practices for improving irrigation in Arid and Semiarid regions. The world Bank, Washington, Technical paper No. 64.

13. Hilgeman, R. H. and F. O. Sharo. 1970. Response of irrigation on yield and quality of orange. Agric. Res. Rev. Cairo, 95: 739-49.

14. Hussein, S. M. 1998. Influence of irrigation levels on the growth, mineral content and fruit quality of "Anna" apple. M. Sci. Thesis, Cairo Univ.

15. Hussein, S. M. M. and T. A. Eid. 2013. Response of plum trees grown in clay loamy soil to the change from surface to drip irrigation system. Agric. J. of Appl. Sci., 28 (5): 218240.

16. Ibrahim, M. A. F. 2001. The effect of different water regimes on morphological, physiological and anatomical characteristics of some citrus rootstocks. Ph. D. Thesis, Fac. of Agric., Cairo Univ., Egypt.

17. Jensen, M.E. 1983. Design and operation of farm irrigation systems. Amer. Soc. Agric. Eng. Michigan, USA, p. 827.

18. Koo, R.C.J. and A.G. Smajstrla. 1985. Trickle irrigation of citrus on sandy soils in a humid region. Drip trickle irrigation in action I. 212-219.

19. Levin, I., R. Assaf and B. A. Bravdo. 1980. Irrigation water status and nutrient uptake in an apple orchard. Butterworths, Borough Green UK p.230.

20. Michael, A.M. 1978. Irrigation theory and practice. Vikas Publishing House PVT LTD New Delhi, Bombay.

21. Naguib, M. M. 1978. Physiological factors affecting creasing of orange fruit. Ph.D. Ain Shams University, Cairo, Egypt.

22. Snedecor, G. W. and W. G. Cochran. 1990. Statistical methods. Oxford and J.B.H. Publishing Com. $7^{\text {th }}$ Edition. 


\section{تأثير تغيير نظام الرى السطحى إلى نظام الرى الحديث (البيلر)}

\section{على أثجار البرتقال أبو سرة المثرة}

شعبان محمد حسين1، السيد عبد الله إسماعيل1، محمد نجيب حسن إسماعيل 1طارق أحمد عيد²

$$
\begin{aligned}
& \text { ا . معرج بحوث البساتين - مركز البحوث الزر/عية - الجيزة - مصر }
\end{aligned}
$$

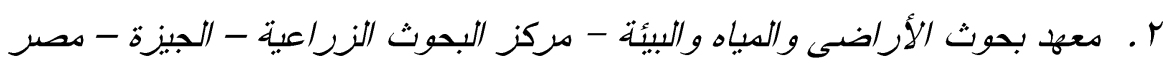

أجريت تجربة حقلية بمزرعة خاصة بقرية وردان بمحافظة الجيزة خلال عامي 2011

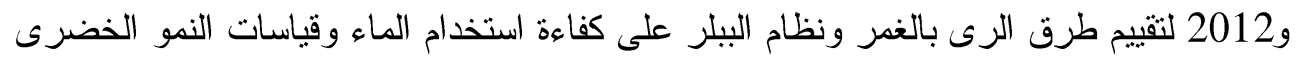
و الجذور وقياسات الإثمار وجودة الثمار ومحتوى الأوراق من العناصر لأشجار البرتقال أبو سلإلإلغة

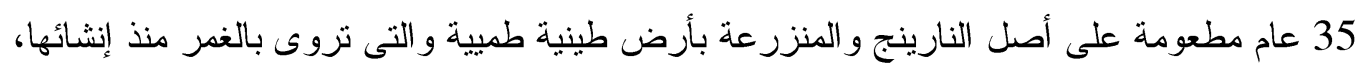

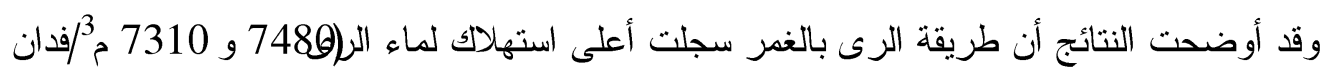

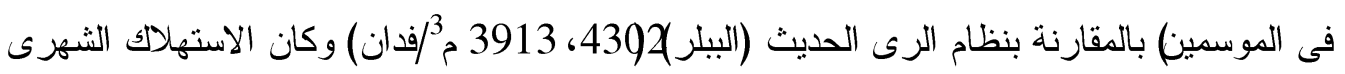

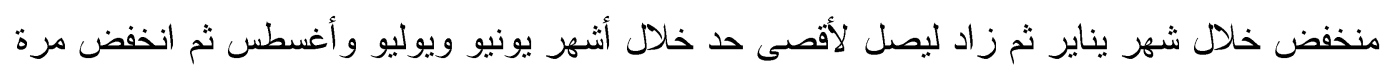
أخرى.

أفادت النتائج أن نظام الرى الببلر أدى إلى زيادة معنوية فى قياسات النمو الخضرى (طول

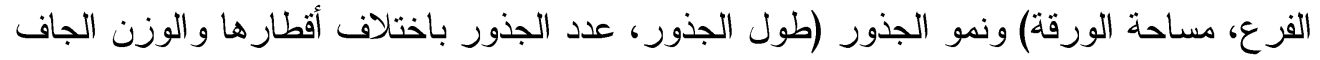

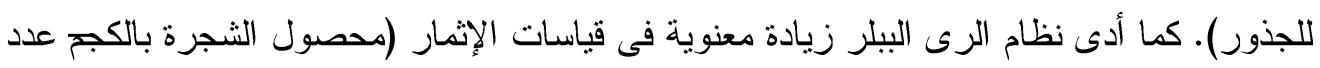

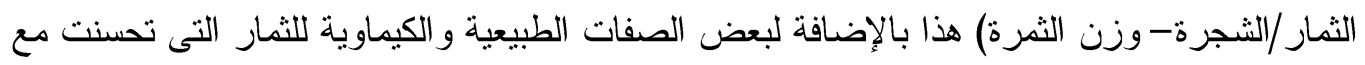

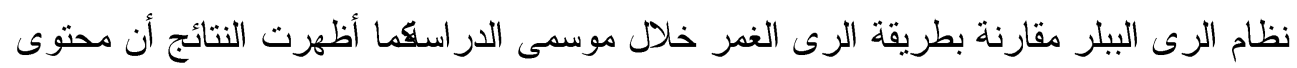

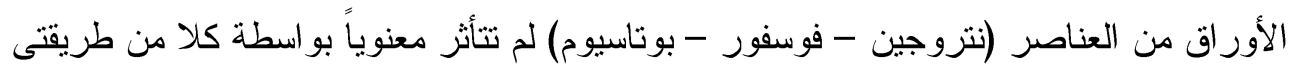

$$
\text { الرى تحت الدر اسة خلال الموسمين. }
$$

ويمكن التوصية بتغيير طريقة الرى الغمر إلى نظام الرى الببلر لأشجار البرتقال أبو سرة

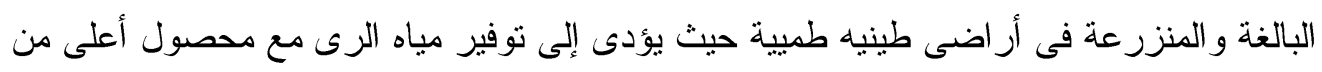

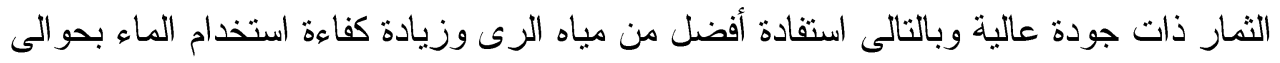

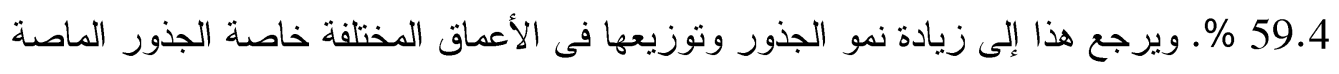

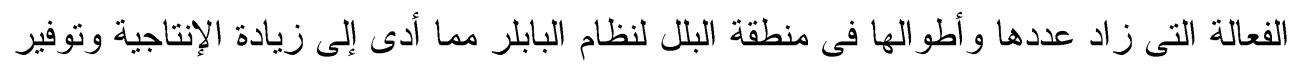

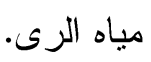

